\title{
FIELD THEORY FOR FUNCTION FIELDS OF SINGULAR PLANE QUARTIC CURVES
}

\author{
KeI MiURA
}

\begin{abstract}
We study the structure of function fields of plane quartic curves by using projections. Taking a point $P \in \mathbb{P}^{2}$, we define the projection from a curve $C$ to a line $l$ with the centre $P$. This projection induces an extension field $k(C) / k\left(\mathbb{P}^{1}\right)$. By using this fact, we study the field extension $k(C) / k\left(\mathbb{P}^{1}\right)$ from a geometrical point of view. In this note, we take up quartic curves with singular points.
\end{abstract}

\section{INTRODUCTION}

Let $k$ be an algebraically closed field of characteristic zero. We fix $k$ as the ground field of our discussion. Let $K$ be an algebraic function field in one variable over $k$. We would like to look into $K$, in particular to see what intermediate fields exist. For this purpose we develop a field theory for $K$.

Let $C$ be an irreducible plane curve of degree $d(d \geqslant 1)$ and $k(C)$ be a rational function field of $C$. Then $K$ is expressed as $K=k(C)$. In this paper we study $K=k(C)$ from a geometrical viewpoint as we have done in $[3]$.

Let $\varepsilon: X \rightarrow C$ be the birational morphism from the smooth model $X$ onto $C$. Then $K=k(C)=k(X)$. Take a point $P \in \mathbb{P}^{2}$. The morphism $\pi_{P}: X \rightarrow \mathbb{P}^{1}$ is the rational function on $X$ defined by

$$
\pi_{P}: X \ni R \longmapsto \overline{P \varepsilon(R)} \in \mathbb{P}^{1}
$$

where $\overline{P \varepsilon(R)}$ is the line passing through $P$ and $\varepsilon(R), \mathbb{P}^{1}$ is the one-dimensional projective space of all lines in $\mathbb{P}^{2}$ passing through $P$. The degree of $\pi_{P}$ is clearly $d-m_{P}$, where $m_{P}$ is the multiplicity of $C$ at $P$. (We put $m_{P}=0$, if $P \notin C$.) Then we have a field extension $\pi_{P}^{*}: k\left(\mathbb{P}^{1}\right) \hookrightarrow K$. This field extension depends only on the point $P$. So we denote the function field $k\left(\mathbb{P}^{1}\right)$ by $K_{P}$, that is,

$$
\pi_{P}^{*}: K_{P} \hookrightarrow K
$$

Received 21st September, 1999

The author would like to express his gratitude to Professor Hisao Yoshihara for suggesting this research and giving valuable advice.

Copyright Clearance Centre, Inc. Serial-fee code: 0004-9727/00 \$A2.00+0.00. 
REMARK 1. The above function field $K_{P}$ is not necessarily a maximal rational subfield of $K$. However if $C$ is a smooth plane curve of degree $d(d \geqslant 2)$ and $P \in \mathbb{P}^{2}$ is a point, then $K_{P}$ is a maximal rational subfield (see [3]).

Note that every subfield $K^{\prime}$ satisfying $k \neq K^{\prime} \subset K_{P}$ is rational by Lüroth's theorem. We are interested in the structure of the field extension $K / K_{P}$. For example,

(1) When is the extension Galois?

(2) Let $L_{P}$ be the Galois closure of $K / K_{P}$. What can we say about $L_{P}$ ?

(3) What is the Galois group $\mathrm{Gal}\left(L_{P} / K_{P}\right)$ ?

(4) How many fields do there exist between $K$ and $K_{P}$ ?

Definition 1. The point $P \in \mathbb{P}^{2}$ is called a Galois point if $K / K_{P}$ is a Galois extension.

Let $\widetilde{C}_{P}$ be the smooth curve with the function field $L_{P}$ and $\tilde{\pi}_{P}: \widetilde{C}_{P} \rightarrow X$ be the covering map induced by $L_{P} \supset K$. We denote the composite map $\pi_{P} \circ \tilde{\pi}_{P}$ by $\theta_{P}$, that is, $\theta_{P}: \tilde{C}_{P} \rightarrow \mathbb{P}^{1}$. It is clear that $\theta_{P}$ is Galois. We call $\tilde{C}_{P}$ the minimal splitting curve of $\pi_{P}: X \rightarrow \mathbb{P}^{\mathbf{1}}$, after Tokunaga [8].

Definition 2. We denote by $G_{P}$ the Galois group $\operatorname{Gal}\left(L_{P} / K_{P}\right)$ and by $g(P)$ the genus of $\widetilde{C}_{P}$.

When $C$ is smooth, we have studied questions (1), (2), (3) and (4) for the case $d=4$ and quintic Fermat curve (see [3, 4]). In what follows we assume that $C$ has at least one singular point. In the case $d=1,2$ or 3 , the above questions are trivial. In this paper we study the questions in the case $d=4$. So henceforth we denote by $C$ a plane quartic curve which is not smooth.

REMARK 2. For a singular point $P \in C$, we have that $\operatorname{deg} \pi_{P}=1$ or 2 . Hence we see that a singular point of $C$ becomes a Galois point. We call this a non-smooth Galois point. In particular, we call the Galois point $P \in \mathbb{P}^{2}$ with $m_{P}=0$ or 1 a smooth Galois point.

Definition 3. We denote the number of smooth Galois points by $\delta(C)$.

We use the following notation:

$f(x, y)=0:$ the defining equation of (the affine part of) $C$.

$f_{i}(x, y)$ : the homogeneous part of degree $i$ of $f(x, y)$.

$g=g(X):$ the genus of $X$.

$m_{P}(C)=m_{P}$ : the multiplicity of $C$ at $P$. (We put $m_{P}=0$, if $P \notin C$.)

$s_{P}(C)=s_{P}$ : the number of the analytic branches of $C$ at $P$.

$I_{P}\left(C_{1}, C_{2}\right):$ the intersection number of $C_{1}$ and $C_{2}$ at $P$.

$W=W(C):$ the sum of order of flex of $C$, that is,

$$
W(C)=\sum_{Q \in C}\left\{I_{Q}\left(C, T_{Q}\right)-2\right\}
$$


where $T_{Q}$ denotes the tangent line to $C$ at $Q \in C$.

Let $\theta_{P}: \widetilde{C}_{P} \rightarrow \mathbb{P}^{1}$ be the Galois covering. Then we define a branch type of $\theta_{P}$ as follows (see [6]). Let $B_{\theta_{P}}=m_{1} Q_{1}+\cdots+m_{d} Q_{d}\left(2 \leqslant m_{1} \leqslant \cdots \leqslant m_{d}\right)$ be the branch locus of $\theta_{P}$. Here $m_{i}$ is called the ramification index of $\theta_{P}$ at $Q_{i}$. That is, if $R$ is a point of $\theta_{P}^{-1}\left(Q_{i}\right)$, then there are local coordinate systems $\zeta$ and $\eta$ around $R$ and $Q_{i}$ respectively with $\zeta(R)=0$ and $\eta\left(Q_{i}\right)=0$ such that $\theta_{P}$ is locally given as: $\zeta \mapsto \eta=\zeta^{m_{i}}$. We say $\theta_{P}$ has branch type $\left(m_{1}, \ldots, m_{d}\right)$ if $B_{\pi_{P}}=m_{1} Q_{1}+\cdots+m_{d} Q_{d}$.

\section{Statement of Results}

Under the situation above the main results are as follows. We state our results separately according to the case $P \in C$ or $P \notin C$.

In the case $P \in C$, we have the following:

TheOREM 1. Suppose $C$ has a triple point. Then we have $g(P)=0$ or 1 for any $P \in C$. If $C$ has a tacnode-cusp, then $C$ has no smooth Galois point.

Theorem 2. Suppose the singularities of $C$ are at most double points. Then for any $C$ and for any $P \in C$, we have that $g(P)=g$ or $3 g+1-a,(0 \leqslant a \leqslant g+1)$. If $P$ is a general point of $C$, then $G_{P}$ is isomorphic to $S_{3}$; the symmetric group on three letters, and $g(P)=3 g+1$.

In the case $P \notin C$, we have the following:

ThEOREM 3. For any plane quartic $C$ and any point $P \in \mathbb{P}^{2} \backslash C, G_{P}$ is isomorphic to one of the following: (1) $S_{4}$; the symmetric group on four letters, (2) $A_{4}$; the alternating group on four letters, (3) $D_{4}$; the dihedral group of order eight, (4) $C_{4}$; the cyclic group of order four or (5) $V_{4}$; Klein's four group. Furthermore, if $C$ has no simple cusp of multiplicity three and $P \in \mathbb{P}^{2} \backslash C$ is a general point, then $G_{P} \cong S_{4}$ and $g(P)=12 g+13$.

Corollary 4. For a general point $P \in \mathbb{P}^{2}$, there exists no field between $K$ and $K_{P}$.

On Galois points, we have the following.

THEOREM 5. If $C$ is a plane quartic curve of genus two (that is, $g(X)=2$ ), then there is no point $P \in \mathbb{P}^{2} \backslash C$ satisfying $G_{P} \cong C_{4}$.

\section{Proof of Theorem 1 AND 2}

First, we consider the ramification points of $\pi_{P}: X \rightarrow \mathbb{P}^{1}\left(P \in \mathbb{P}^{2}\right)$. From the definition of $\pi_{P}$, we see that the following assertions hold true.

(i) If $Q$ is a smooth point of $C$ :

Then there exist $\widetilde{Q} \in X$ such that $\varepsilon(\widetilde{Q})=Q$. Hence we have that the ramification index of $\pi_{P}$ at $\tilde{Q}$ (which we denote by $e_{\tilde{Q}}$ ) equals $I_{Q}(C, \overline{P Q}$ ). For example, if $\overline{P Q}$ is the tangent line at $Q$, then $e_{\tilde{Q}} \geqslant 2$. 
(ii) If $Q$ is a singular point of $C$ :

Let $C_{1}, C_{2}, \cdots, C_{s}$ be the analytic branches at $Q$, and $\varepsilon^{-1}(Q)=\tilde{Q}_{1}, \cdots, \widetilde{Q}_{s}$, where $s=s_{Q}(C)$. Then we have a one-to-one correspondence between $C_{j}$ and $\tilde{Q}_{j}$.

If $\overline{P Q}$ is not a tangent line to $C_{j}$, then $e_{\tilde{Q}_{j}}=m_{Q}\left(C_{j}\right)=I_{Q}\left(C_{j}, \overline{P Q}\right)$.

If $\overline{P Q}$ is a tangent line to some $C_{k}$, then $e_{\tilde{Q}_{k}}=I_{Q}\left(C_{k}, \overline{P Q}\right)$.

REMARK 3. From the Riemann-Hurwitz formula for $\pi_{P}\left(P \in \mathbb{P}^{2}\right)$, we have

$$
\sum_{R \in X}\left(e_{R}-1\right)=2 g(X)+6-2 m_{P} .
$$

We prove Theorems 1 and 2. For a singular point $P \in C$, the above questions (1) (4) are trivial (see Remark 2). So henceforth we assume that $P \in C$ is a non-singular point of $C$, that is, $m_{P}=1$.

By taking a suitable set of coordinates, we can assume that

(i) $P=(0,0)$,

(ii) $y=0$ is the tangent line to $C$ at $P$,

(iii) the singular points of $C$ do not lie on $x=0$,

(iv) $x=0$ and $C$ meet transversally,

(v) if $l$ is a line passing through $P$ and a point of $C$ at infinity, then $l$ is not a tangent line to $C$ and $l$ does not pass through the singular points of $C$.

Let $l_{t}$ be the line $y=t x$. Then we may assume that the projection is defined as $\pi_{P}\left(C \cap l_{t}\right)=t$, if $l_{t}$ does not pass through the singular points of $C$. In the affine plane $(x, t) \in \mathbb{A}^{2}$, let $\widehat{C}$ be the curve defined by

$$
\widehat{f}(x, t)=f(x, t x) / x=\varphi_{4}(t) x^{3}+\varphi_{3}(t) x^{2}+\varphi_{2}(t) x+\varphi_{1}(t),
$$

where $\varphi_{i}(t)=f_{i}(1, t)(1 \leqslant i \leqslant 4)$. Then $K=k(x, t)$ and $K_{P}=k(t)$, the extension $K / K_{P}$ is obtained by $\widehat{f}(x, t)=0$. (When $t=\infty$, we consider $x=s y$ instead, where $s t=1$. Indeed, $\widehat{C}$ is an affine part of the blow-up of $C$.) Then we may study $\pi_{P}: X \rightarrow \mathbb{P}^{1}$ by considering a projection from $\widehat{C}$ to the $t$-axis.

We can find the branch points of $\pi_{P}$ by using the discriminant of $\hat{f}(x, t)$. Let $\psi(t)$ be the discriminant of $\hat{f}(x, t) \in k[t][x]$, that is,

$$
\psi(t)=\left(\varphi_{4}(t)\right)^{4} \prod_{i<j}\left(x_{i}-x_{j}\right)^{2}
$$

where $x_{i}$ are the roots of $\hat{f}(x, t)=0$ in $\overline{k(t)}$.

Definition 4. The point $Q \in C$ is called a 1 -flex [respectively 2-flex], if $I_{Q}\left(C, T_{Q}\right)=3$ [respectively 4], where $T_{Q}$ is the tangent line to $C$ at $Q$. 
Noting that $I_{P}\left(C, T_{P}\right)=I_{\hat{P}}\left(\widehat{C}, T_{\hat{P}}\right)+1$, where $T_{P}$ is the tangent line to $C$ at $P \in C$, we see that $\widehat{P}$ is the intersection point of $\widehat{C}$ and $t=0$. We have the following lemmas by copying the proof of [3, Lemma 3.2].

LEMma 1. The discriminant $\psi(t)$ is expressed as $\psi(t)=\psi_{0}(t) \psi_{1}(t)$. If $(t-\alpha)^{n}$ is a factor of $\psi_{0}(t)$, then we have $n=1,2$. Suppose that $\alpha \neq 0$. Then $n=2$ [respectively $n=1$ ] if and only if the line $l_{\alpha}$ becomes a tangent line to $C$ at a 1-flex [respectively not a flex]. On the contrary, suppose that $\alpha=0$. Then $n=2$ if and only if $P$ is a 2-flex, $n=1$ if and only if $P$ is a 1 -flex or $l_{0}$ is a bitangent line.

LEMMA 2. Let $P_{i}=\left(a_{i}, b_{i}\right)$ be the singular points of $C(1 \leqslant i \leqslant r)$. If $(t-\beta)^{m}$ is a factor of $\psi_{1}(t)$, then the line $y=\beta x$ passes through some $P_{i}$.

REMARK 4. It is well-known that

$$
-\varphi_{4}(t) \psi(t)=\operatorname{Res}(\widehat{f}, \partial \widehat{f} / \partial x),
$$

where $\operatorname{Res}(\widehat{f}, \partial \hat{f} / \partial x)$ is the resultant of $\widehat{f}$ and $\partial \hat{f} / \partial x$ with respect to $x$. By our coordinates condition (v), if $\varphi_{4}(\alpha)=0$, then $t=\alpha$ is not a branch point. Hence we have $m=\sum I_{\widehat{Q}_{i}}\left(\widehat{C}, \widehat{C}_{x}\right)$, where $\left\{\widehat{Q}_{1}, \cdots, \widehat{Q}_{q}\right\}=\widehat{C} \cap\{t=\beta\}$, and $\widehat{C}_{x}$ denotes the curve defined by $\partial \hat{f} / \partial x$.

We call $\psi_{0}(t)$ the smooth part of $\psi(t)$, and $\psi_{1}(t)$ the singular part of $\psi(t)$. In particular, we can find flexes and singular points of $C$ by computing the resultant of $\widehat{f}(x, t)$ and $\partial \widehat{f}(x, t) / \partial x$.

Next, suppose that $P \in C$ is not a Galois point. Then we consider the branch points of $\widetilde{\pi}_{P}: \widetilde{C}_{P} \rightarrow X$. Referring to [8], we have the following proposition.

Proposition 6. Let $\Delta\left(X / \mathbb{P}^{1}\right)$ and $\Delta\left(\widetilde{C}_{P} / \mathbb{P}^{1}\right)$ be the branch loci of $\pi_{P}$ and $\theta_{P}$ respectively. Then we have $\Delta\left(X / \mathbb{P}^{1}\right)=\Delta\left(\widetilde{C}_{P} / \mathbb{P}^{1}\right)$.

Hence we have the following lemma.

Lemma 3. A point $Q \in X$ is a branch point of $\tilde{\pi}_{P}$ if and only if the following conditions are satisfied:

(a) suppose $\pi_{P}(Q)=\alpha$, then $\pi_{P}^{-1}(\alpha)=\left\{Q, Q^{\prime}\right\}$,

(b) $\pi_{P}$ has ramification index one at $Q$, two at $Q^{\prime}$.

PROOF: Note that the Galois covering has the same ramification indices at each branch point. Suppose $R$ is a branch point of $\tilde{\pi}_{P}$ such that the ramification index of $\pi_{P}$ at $R$ is three. Put $\pi_{P}(R)=\beta$. Then $\theta_{P}$ has ramification index six at $t=\beta$. Since $S_{3}$ does not contain the cyclic group of order six as a subgroup, this is a contradiction. Next, $R^{\prime}$ is a branch point of $\tilde{\pi}_{P}$ such that the ramification index of $\pi_{P}$ at $R^{\prime}$ is two. Put $\pi_{P}\left(R^{\prime}\right)=\gamma$. Then $\theta_{P}$ has ramification index four at $t=\gamma$. Since the degree of $\theta_{P}$ is six, this is a contradiction. Hence by Proposition 6, this proves the lemma.

Furthermore we have the following. 
LEMma 4. If $P$ is not a Galois point, then $g(P)=3 g(X)+1-a,(0 \leqslant a \leqslant$ $g(X)+1)$.

PROOF: Let $a$ and $b$ be the numbers of ramification points of $\pi_{P}$ whose ramification indices are three and two respectively. By Lemma 3, the number of branch points of $\tilde{\pi}_{P}$ $: \widetilde{C}_{P} \rightarrow X$ equals $b$. By the Riemann-Hurwitz formula for $\tilde{\pi}_{P}$, we have that $2 g(P)-2=$ $2(2 g(X)-2)+b$. By Remark 3, we have that $2 a+b=2 g(X)+4$. Hence we obtain that $g(P)=3 g(X)+1-a$. However, if $b=0$, then $\pi_{P}$ is a Galois covering by $[8$, Proposition 3.1]. Thus $a$ can not be $g(X)+2$. This proves the lemma.

REMARK 5. Referring to [8], we have the following assertion. A point $P$ is a smooth Galois point if and only if $b=0$. Hence $G_{P} \cong S_{3}$ if and only if $b \neq 0$.

Suppose $C$ has a tacnode-cusp $Q$. Then we have that $m_{Q}=3$ and $s_{Q}=2$. Hence we infer that $\pi_{P}$ always has a ramification point with ramification index two for any $P \in C$. Indeed since the line $\overline{P Q}$ is not tangent at $Q$, we have

$$
\sum_{R \in \varepsilon^{-1}(Q)}\left(e_{R}-1\right)=m_{Q}-s_{Q}=1
$$

By the above remark, we see that $C$ has no smooth Galois point.

If $C$ has a simple cusp of multiplicity three, then for any $P \in C$, there exist a line passing through $P$ which meets $C$ at the cusp with intersection number three. Hence we infer that $\pi_{P}$ always has a ramification point with ramification index three. Therefore we infer the following.

LEmma 5. Suppose $C$ has no simple cusp of multiplicity three. Then $a=0$ for a general point $P \in C$.

Combining the above results, we obtain the assertions in Theorem 1 and 2.

REMARK 6. Suppose $C$ has an ordinary triple point $Q$ and the line $l_{\alpha}$ passes through $P$ and $Q$. Then the line $l_{\alpha}$ passes through $Q$ with the intersection number three, and $\varepsilon^{-1}(Q)$ consists of three points in $X$. So we see that $\pi_{P}$ is unramified over $t=\alpha$.

Next, as an example, we consider the curve $C$ defined by $y+g(x, y)=0$, where $g(x, y)$ is a homogeneous polynomial of degree four and $g(x, 0) \neq 0$.

Claim 1. The curve $C$ has a smooth Galois point $P=(0,0)$.

Proof: Putting $f(x, y)=y+g(x, y)$, we have $\hat{f}(x, t)=g(1, t) x^{3}+t$. Since the field extension $K / K_{P}$ is given by $x^{3}=-t / g(1, t)$, the claim is clear.

Then the homogeneous equation of $C$ is $F(x, y, z)=y z^{3}+g(x, y)$, where $x, y, z$ are the homogeneous coordinates of $\mathbb{P}^{2}$.

Claim 2. The singular points of $C \subset \mathbb{P}^{2}$ exist only on the line $z=0$, and satisfy $\partial g / \partial x=\partial g / \partial y=0$. 
Proof: Since $C \subset \mathbb{P}^{2}$ is defined by $F(x, y, z)=0$, the claim is clear by considering $\partial F / \partial x=\partial F / \partial y=\partial F / \partial z=0$.

Then we may assume that $g(x, y)$ is the one of the following:

(i) $g(x, y)=(y-\alpha x)^{2}(y-\beta x)(y-\gamma x)$,

(ii) $g(x, y)=(y-\alpha x)^{2}(y-\beta x)^{2}$,

(iii) $g(x, y)=(y-\alpha x)^{3}(y-\beta x)$,

(iv) $g(x, y)=(y-\alpha x)^{4}$,

where $\alpha, \beta, \gamma$ are mutually distinct elements of $k \backslash\{0\}$. Then the singular points of $C$ for each case are the following:

(i) $(1: \alpha: 0)$ is the only singular point and is a simple cusp of multiplicity two,

(ii) $(1: \alpha: 0)$ and $(1: \beta: 0)$ are the only singular points and are simple cusps of multiplicity two,

(iii) $(1: \alpha: 0)$ is the only singular point and is an ordinary triple point,

(iv) $(1: \alpha: 0)$ is the only singular point and is a simple cusp of multiplicity three.

In case (i), we see that $(1: \beta: 0)$ and $(1: \gamma: 0)$ are 1 -flexes, and the line $y=\beta x$ [respectively $y=\gamma x$ ] is the tangent line at $(1: \beta: 0)$ [respectively $(1: \gamma: 0)$ ]. Of course $P$ is a 2-flex. Furthermore the line $y=\alpha x$ is the tangent line at $(1: \alpha: 0)$. Hence $\pi_{P}$ has branch type $(3,3,3,3)$. Indeed $\pi_{P}$ has ramification index three at $t=\alpha, t=\beta, t=\gamma$ and $t=0$.

In case (ii), we see that $\pi_{P}$ has branch type $(3,3,3)$ by an argument similar to (i). In particular, in cases (i) and (ii), there are no more smooth Galois points (by considering the branching data of the other points). Indeed for an other point $Q \in C(Q \neq P)$ and a line $l_{\lambda}$ passing through $Q$, we see that $l_{\lambda}$ meets $C$ at $(1: \alpha: 0)$ with intersection number two and it intersects $C$ transversally at the other point. Hence $\pi_{Q}^{-1}(\lambda)$ is a two point, so we see $\pi_{Q}$ is not Galois.

In case (iii), we see that $\pi_{P}$ has branch type $(3,3)$. Indeed $\pi_{P}$ has ramification index three at $t=0$ and $t=\beta$.

In case (iv), we see that $\pi_{P}$ has branch type $(3,3)$. Referring to [1], we have $W(C)=$ 2. Since $P$ is a 2 -flex, there is no more flex. Hence we have $\delta(C)=1$ in cases (i), (ii) and (iv).

\section{Proof of Theorem 3 and 5}

Next we consider the case $P \in \mathbb{P}^{2} \backslash C$. By taking a suitable set of coordinates, we may assume

(i) $P=(0,0) \notin C$, 
(ii) the singular points of $C$ do not lie on $x=0$,

(iii) $x=0$ and $C$ meet transversally.

Let $l_{t}$ be the line $y=t x$. Then we may assume that the projection is defined as $\pi_{P}\left(C \cap l_{t}\right)=t$, if $l_{t}$ does not pass through the singular points of $C$. In the affine plane $(x, t) \in \mathbb{A}^{2}$, let $\check{C}$ be the curve defined by

$$
\check{f}(x, t)=f(x, t x) / x=\varphi_{4}(t) x^{4}+\varphi_{3}(t) x^{3}+\varphi_{2}(t) x^{2}+\varphi_{1}(t) x+c,
$$

where $\varphi_{i}(t)=f_{i}(1, t)(1 \leqslant i \leqslant 4)$ and $c$ is a non-zero element of $k$. Then $K=k(x, t)$ and $K_{P}=k(t)$, the extension $K / K_{P}$ is obtained by $\check{f}(x, t)=0$. We can find flexes and singular points of $C$ by an argument similar to that in Lemmas 1 and 2 . So we know the ramification points of $\pi_{P}: X \rightarrow \mathbb{P}^{\mathbf{1}}$. We use $\psi(t), \psi_{0}(t)$ and $\psi_{1}(t)$ as in Lemmas 1 and 2 .

Lemma 6. Suppose $C$ has no simple cusp of multiplicity three. If $P$ is a general point, then $\pi_{P}$ has just $2 g(X)+6$ ramification points, and its ramification indices are two.

Proof: If $P$ is a general point and $l$ is a line passing through $P$, then one of the following assertions holds true:

(a) The line $l$ intersects $C$ transversally.

(b) The line $l$ touches at one point $Q \in C$ with $I_{Q}(C, l)=2$, and it intersects $C$ transversally at the other points.

(c) The line $l$ is not a tangent line at singular points.

Hence we prove the lemma from the Riemann-Hurwitz formula for $\pi_{P}$.

Let $P$ be a general point and $\theta_{P}: \widetilde{C}_{P} \rightarrow \mathbb{P}^{1}$ be the Galois covering. If $R \in \widetilde{C}_{P}$ is a ramification point, then we infer that the ramification number of $\theta_{P}$ at $R$ is two by the above lemma. Now the theorem is proved by copying after the proof of $[7$, Theorem 4.4.5]. Since $\theta_{P}$ is unramified over $t=\infty$ and the inertia group at the ramification point $R$ is generated by a transposition, the latter part of the theorem is a consequence of $[\mathbf{7}$, Proposition 4.4.6 and Lemma 4.4.4]. From the Riemann-Hurwitz formula, we obtain that $2 g(P)-2=24(0-2)+12(2 g(X)+6)$. Hence we have $g(P)=12 g(X)+13$. Corollary 4 is a general fact of Galois theory and the structure of $S_{4}$.

Now we define the cubic resolvent $\tilde{g}(z)$ of $\check{f}(x, t)=0$ as follows:

$$
\widetilde{g}(z)=z^{3}-\frac{\varphi_{2}}{\varphi_{4}} z^{2}+\frac{\varphi_{1} \varphi_{3}-4 c \varphi_{4}}{\varphi_{4}^{2}} z+\frac{4 c \varphi_{2} \varphi_{4}-c \varphi_{3}^{2}-\varphi_{1}^{2} \varphi_{4}}{\varphi_{4}^{3}},
$$

where $\varphi_{i}=\varphi_{i}(t)(1 \leqslant i \leqslant 4)$. Clearly $\widetilde{g}(z) \in k(t)[z]$. Then we have the following facts from field theory (see [2]).

FACT 7. Let $M$ be the splitting field of $\widetilde{g}(z)=0$ over $k(t)$. Then we have the following. 
(i) $G_{P} \cong S_{4} \Leftrightarrow \tilde{g}(z)$ is irreducible over $k(t)$ and $\sqrt{\psi(t)} \notin k(t)$.

(ii) $G_{P} \cong A_{4} \Leftrightarrow \widetilde{g}(z)$ is irreducible over $k(t)$ and $\sqrt{\psi(t)} \in k(t)$.

(iii) $G_{P} \cong V_{4} \Leftrightarrow \tilde{g}(z)$ splits into linear factors over $k(t)$.

(iv) $G_{P} \cong C_{4} \Leftrightarrow \tilde{g}(z)$ has exactly one root $\alpha$ in $k(t)$ and $h(x):=\left(x^{2}-\alpha x+\right.$ $\left.c / \varphi_{4}\right)\left(x^{2}+\left(\varphi_{3} / \varphi_{4}\right) x+\varphi_{2} / \varphi_{4}-\alpha\right)$ splits over $M$.

(v) $G_{P} \cong D_{4} \Leftrightarrow \widetilde{g}(z)$ has exactly one root $\alpha$ in $k(t)$ and $h(x)$ does not split over $M$.

Next, we would like to characterise the defining equations by the structure of $G_{P}$. First, we recall general facts in field theory.

FACT 8. If $G_{P}$ is isomorphic to $S_{4}$ or $A_{4}$, then there exists no field between $K$ and $K_{P}$.

In the cases when $G_{P} \cong V_{4}, C_{4}$ or $D_{4}$, we can find subfields between $K$ and $K_{P}$ by considering the subgroups of $G_{P}$. The following lemma is clear.

LEMMA 7. The following assertions are equivalent.

(a) $K$ contains an intermediate subfield $K^{\prime}$ with $\left[K^{\prime}: K_{P}\right]=2$.

(b) $K$ is expressed as $K=K_{P}(\xi)$, where $\xi$ satisfies an irreducible polynomial $x^{4}+a x^{2}+b \in K_{P}[x]$.

From the above lemma, we obtain that $K=k(x, t)$, where $x^{4}+a x^{2}+b=0$. The coefficients $a$ and $b$ are in $k(t)$, so we denote these by $a(t), b(t)$ respectively. By cancelling the denominator, we obtain $c(t) x^{4}+d(t) x^{2}+e(t)=0$, where $c(t), d(t)$ and $e(t) \in k[t]$, and they are assumed to be relatively prime. Putting $y=t x$, we obtain that

$$
c(y / x) x^{4}+d(y / x) x^{2}+e(y / x)=0 .
$$

In order to obtain a quartic equation, $\operatorname{deg} e(t)$ must be zero, $\operatorname{deg} d(t) \leqslant 2$ and $\operatorname{deg} c(t) \leqslant 4$. Whence we infer the following assertion. The group $G_{P}$ is isomorphic to $V_{4}, C_{4}$ or $D_{4}$ if and only if the quartic curve $C$ is birationally equivalent to the curve defined by $f_{4}(x, y)+f_{2}(x, y)+c=0$, where $f_{i}(x, y)$ is a homogeneous polynomial of degree $i(i=2,4)$, and $c$ is a non-zero element of $k$. Then we have that $\check{f}(x, t)=\varphi_{4}(t) x^{4}+\varphi_{2}(t) x^{2}+c$ and $\tilde{g}(z)=\left(z-\varphi_{2} / \varphi_{4}\right)\left(z^{2}-4 c / \varphi_{4}\right)$. Applying Fact 1 to this case, we have the following:

(i) $G_{P} \cong V_{4} \Leftrightarrow \sqrt{c / \varphi_{4}} \in k(t)$.

(ii) $G_{P} \cong C_{4} \Leftrightarrow \sqrt{c / \varphi_{4} \cdot\left(\varphi_{2}^{2} / \varphi_{4}^{2}-4 c / \varphi_{4}\right)} \in k(t)$.

(iii) $G_{P} \cong D_{4} \Leftrightarrow$ neither (i) nor (ii).

By using this fact, we present some examples. 
EXAMPLE 1. If $C$ is the curve defined by $x^{4}-2 x^{2} y^{2}+y^{4}+x^{2}+y^{2}+1=0$ and $P=(0,0)$, then we have that $G_{P} \cong V_{4}$ and $g(X)=1$. Indeed we have

$$
\begin{aligned}
\check{f}(x, t) & =\left(t^{4}-2 t^{2}+1\right) x^{4}+\left(t^{2}+1\right) x^{2}+1, \\
\widetilde{g}(z) & =\left(z+\frac{2}{t^{2}+1}\right)\left(z-\frac{2}{t^{2}+1}\right)\left(z-\frac{t^{2}+1}{\left(t^{2}-1\right)^{2}}\right), \\
\psi(t) & =16\left(t^{2}-3\right)^{2}\left(3 t^{2}-1\right)^{2}(t+1)^{2}(t-1)^{2} .
\end{aligned}
$$

The singular points of $C$ are $(1:-1: 0)$ and $(1: 1: 0)$ which are nodes. Since the lines $y= \pm x$ are not tangent lines at these nodes, $\pi_{P}$ is unramified at $t= \pm 1$. Furthermore the line $y=\alpha x$, where $\alpha$ satisfies $\left(\alpha^{2}-3\right)^{2}\left(3 \alpha^{2}-1\right)^{2}=0$, is the bitangent line of $C$. Hence $\pi_{P}$ has branch type $(2,2,2,2)$. By calculating the equation $\check{f}(x, t)=0$, we have that

$$
K=k(x, t)=k\left(t, \sqrt{t^{2}-3}, \sqrt{1-3 t^{2}}\right) .
$$

Then we obtain three intermediate subfields between $K$ and $K_{P}$ by considering the subgroups of $V_{4}$. Indeed we have

$$
K_{1}=k\left(t, \sqrt{1-3 t^{2}}\right), K_{2}=k\left(t, \sqrt{t^{2}-3}\right), \text { and } K_{3}=k\left(t, \sqrt{-3+10 t^{2}-3 t^{4}}\right) .
$$

Let $C_{i}$ be the smooth curve defined by $K_{i}(1 \leqslant i \leqslant 3)$. Then we have that $g\left(C_{1}\right)=0$, $g\left(C_{2}\right)=0$ and $g\left(C_{3}\right)=1$, where $g\left(C_{i}\right)$ is the genus of $C_{i}$.

EXAMPLE 2. If $C$ is the curve defined by $y^{4}-x y^{3}+x^{2}+1=0$ and $P=(0,0)$, then we have that $G_{P} \cong D_{4}$ and $g(X)=2$. Indeed we have

$$
\begin{aligned}
\check{f}(x, t) & =\left(t^{4}-t^{3}\right) x^{4}+x^{2}+1, \\
\psi(t) & =16(t-1)\left(4 t^{4}-4 t^{3}-1\right)^{2} t^{3} .
\end{aligned}
$$

The point $(1: 0: 0)$ is the only singular point of $C$ and it is a simple cusp of multiplicity two. Note that the line $y=\alpha x$ is the bitangent line of $C$, where $\alpha$ satisfies $4 \alpha^{4}-4 \alpha^{3}-1=$ 0 . Furthermore let $x_{i}(1 \leqslant i \leqslant 4)$ be the roots of the equation $\dot{f}(x, t)=0$. We have the following diagram of Galois correspondences:

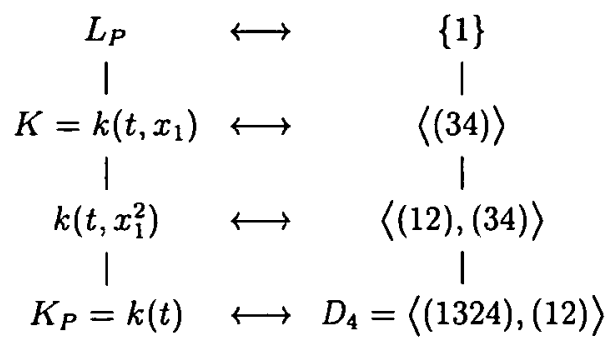

Let $C^{\prime}$ be the smooth curve defined by $k\left(t, x_{1}^{2}\right)$. Then we can check easily that the double covering $C^{\prime} \rightarrow \mathbb{P}^{1}$ branches at four points satisfying $4 t^{4}-4 t^{3}-1=0$, so $g\left(C^{\prime}\right)=1$. Since the function field $L_{P}$ is isomorphic to $k\left(t, x_{1}, \sqrt{\psi(t)}\right)$, we see that $\theta_{P}: \widetilde{C}_{P} \rightarrow \mathbb{P}^{1}$ has branch type $(2,2,2,2,2,2)$, so $g(P)=5$. 
Next, we present an example of a curve $C$ satisfying $G_{P} \cong A_{4}$ for some $P \notin C$. Then, from Fact 1, we infer the following.

REMARK 7 . The group $G_{P} \cong A_{4}$ if and only if the field extension $M / k(t)$ is a cubic Galois extension.

Then we see that $L_{P}=k(t, x, z)$. Let $R_{P}$ be the smooth curve defined by $M=k(t, z)$. Since $k[t, x, z]$ is a tensor product of $k[t, x]$ and $k[t, z]$ over $k[t], \widetilde{C}_{P}$ is the desingularisation of the fibre product of $X$ and $R_{P}$ over $\mathbb{P}^{1}$.

EXAMPLE 3. If $C$ is the curve defined by $x^{4}+x^{3} y+x y^{3}+y^{4}+2 x^{3}+2 y^{3}+2 x+2 y+1=0$ and $P=(0,0)$, then we have that $G_{P} \cong A_{4}$ and $g(X)=2$. Indeed we have

$$
\begin{aligned}
\check{f}(x, t) & =\left(t^{4}+t^{3}+t+1\right) x^{4}+\left(2 t^{3}+2\right) x^{3}+(2+2 t) x+1, \\
\psi(t) & =-432\left(t^{2}-t+1\right)^{2}\left(2 t^{2}+t+2\right)^{2}(t+1)^{4} .
\end{aligned}
$$

The point $(1:-1: 0)$ is the only singular point of $C$ and it is a biflecnode. The line $y=\alpha x$, where $\alpha$ satisfies $\left(\alpha^{2}-\alpha+1\right)^{2}\left(2 \alpha^{2}+\alpha+2\right)^{2}=0$, becomes a tangent line at the 1-flex of $C$, and the line $y=-x$ is a tangent line at the biflecnode. Therefore the number of ramification points of $\pi_{P}$ is five, and its ramification indices are three. Furthermore the field extension $M / k(t)$ is a Galois extension given by $x^{3}=-4\left(2 t^{2}+t+2\right)(t+1)^{2}\left(t^{2}-t-1\right)$. Hence $G_{P} \cong A_{4}$. We obtain a triple Galois covering $R_{P} \rightarrow \mathbb{P}^{1}$, its branch type is $(3,3,3,3,3)$. From the above consideration, we see that $\theta_{P}: \widetilde{C}_{P} \rightarrow \mathbb{P}^{1}$ has branch type $(3,3,3,3,3)$, so $g(P)=9$.

Finally we prove Theorem 5. First we find the conditions when $G_{P} \cong C_{4}$. Since $K / K_{P}$ is a Galois extension, its Galois group is isomorphic to $C_{4}$. Hence $K$ can be expressed as $k(x, t)$ where $x^{4}=a(t) / b(t) \in k(t)=K_{P}$. Putting $y=t x$, we have $b(y / x) x^{4}=a(y / x)$. In order to obtain a quartic equation, $\operatorname{deg} a(t)$ must be zero. Whence we infer the following assertion. The covering $\pi_{P}: X \rightarrow \mathbb{P}^{1}$ is Galois with its Galois group $G_{P} \cong C_{4}$ if and only if the quartic curve $C$ is birationally equivalent to the curve defined by $g(x, y)+c=0$, where $g(x, y)$ is a homogeneous polynomial of degree four and $c$ is a non-zero element of $k$.

Then we study the curve defined by $g(x, y)+c=0$ with $g(x, y)$ and $c$ as above. The homogeneous equation of the curve is $F(x, y, z)=c z^{4}+g(x, y)$, where $x, y, z$ are the homogeneous coordinates of $\mathbb{P}^{2}$. Then we may assume that $g(x, y)$ is the one of the following:

$$
\begin{aligned}
\text { (i) } g(x, y) & =(y-\alpha x)^{2}(y-\beta x)(y-\gamma x) \\
\text { (ii) } g(x, y) & =(y-\alpha x)^{2}(y-\beta x)^{2} \\
\text { (iii) } g(x, y) & =(y-\alpha x)^{3}(y-\beta x) \\
\text { (iv) } g(x, y) & =(y-\alpha x)^{4}
\end{aligned}
$$

where $\alpha, \beta, \gamma$ are mutually distinct elements of $k$. Then the singular points of $C$ for each case are same as the case $P \in C$. But we see that $F(x, y, z)$ is not irreducible in cases 
(ii) and (iv). So we consider cases (i) and (iii).

In case (i), we see that the singular point $(1: \alpha: 0)$ is locally defined by $y^{2}=z^{4}$, and the line $y=\alpha x$ is the tangent line at this point. Furthermore we see that $(1: \beta: 0)$ and $(1 ; \gamma: 0)$ are 2 -flexes. Then we have $g(X)=1$ and the branch type of $\pi_{P}: X \rightarrow \mathbb{P}^{1}$ is $(2,4,4)$. Indeed $\pi_{P}$ has ramification index two at $t=\alpha$ and four at $t=\beta$ and $t=\gamma$.

In case (iii), we see that the singular point $(1: \alpha: 0)$ is a simple cusp of multiplicity three, and the line $y=\alpha x$ is the tangent line at this point. Furthermore we see that $(1: \beta: 0)$ is a 2 -flex. Then we have $g(X)=0$ and the branch type of $\pi_{P}: X \rightarrow \mathbb{P}^{1}$ is $(4,4)$.

In particular, we obtain Theorem 5 . Thus we complete the proofs.

\section{REFERENCES}

[1] S. Iitaka, K. Ueno and Y. Namikawa, Descartes no seishin to Daisûkika, (in Japanese) (Nippon Hyoron Sha, Tokyo, 1980).

[2] L. Kappe and B. Warren, 'An elementary test for the Galois group of a quartic polynomial', Amer. Math. Monthly 96 (1989), 133-137.

[3] K. Miura and H. Yoshihara, 'Field theory for function fields of plane quartic curves', $J$. Algebra 226 (2000), 283-294.

[4] K. Miura and H. Yoshihara, 'Field theory for the function field of the quintic Fermat curve', Comm. Algebra 28 (2000), 1979-1988.

[5] M. Namba, Geometry of projective algebraic curves (Marcel Dekker, New York, Basel, 1984).

[6] M. Namba, Branched coverings and algebraic functions, Pitman Research Notes in Mathematics 161 (Longman, Harlow, 1987).

[7] J.P. Serre, Topics in Galois theory (Notes written by H. Darmon), Research Notes in Mathematics 1 (Jones and Bartlett Publ., Boston, London, 1992).

[8] H. Tokunaga, 'Triple coverings of algebraic surfaces according to the Cardano formula', J. Math. Kyoto Univ. 31 (1991), 359-375.

Graduate School of Science and Technology

Niigata University

Niigata 950-2181

Japan

e-mail: kmiura@scux.sc.niigata-u.ac.jp 